\title{
Design of Precision Fertilization Management Information System on GPS and GIS Technologies*
}

\author{
Zhimin $\mathrm{Liu}^{1,2}$, Weidong Xiong ${ }^{3}$, and Xuewei Cao ${ }^{1}$ \\ ${ }^{1}$ Geomatics College, Shandong University of Science and Technology, \\ Qingdao, China \\ ${ }^{2}$ Key Laboratory of Surveying and Mapping, \\ Technology Shandong Prince, China, 266590 \\ ${ }^{3}$ Survey mapping and planning office, \\ Qingdao Development Zone, Qingdao,China, 266555 \\ liuzhimin010@163.com
}

\begin{abstract}
Aimed on the efficiency, energy-saving, yield and pro-environment, the precision agriculture is needed to develop. Presently, the field information quick-acquisition technology has become the important topics in the international precision agriculture research fields, because that still far behind the development of other precision agriculture technologies. The technological innovations focus on the new surveying technology, such as the fast real-time operation, which are helpful for improving sampling densities and accuracy. The author firstly summarized the three aspects development of data acquisition, data processing and management information system based on GPS and GIS of Precision Fertilization Management Information System (PFMIS), then we designed the framework of PFMIS which included the data-acquisition technique on the continuously operating reference stations (CORS), the dataprocessing methods on intelligence algorithms and Management Information System (MIS) on ArcGIS Engine, in order to satisfy the needs of network publication and query to precision fertilization.
\end{abstract}

Keywords: Precision Fertilization, PFMIS, GPS, GIS, CORS.

\section{Introduction}

In the information society, precision agriculture has become the focus on the frontier forces to how to use rationally the agricultural resources, enhance the quality and quantity of the agricultural production, reduce the production costs, and improve the ecological environment and sustainable agricultural development in the international developed country in the 21 st century [1]. Based on the information and knowledge to the agricultural production management systems, the practice to this new concept would have the revolutionary significance, that promote the transformation of the information technology application, and enhance the civil traditional agriculture level

\footnotetext{
* Sponsored by the National Natural Science Foundation of China (40704001, 40876051).
} 
and agricultural equipment technology, develop the research and application of agricultural production-oriented of information technology, and achieve sustainable agricultural development [2,3]. Food shortage and fertilizer price-rising is a global issue. And with the multifaceted problems on national economy of the impact on food security, environmental pollution, lack of resources, increased costs, the precision fertilizer management information systems (PFMIS) would be established, that included the spatial data collection, soil nutrient data test, data standardization, management information system platform, the expert systems of the fertilizer recipes and fertilization model, and network queries, in order to achieve "increased food production and fertilizer efficiency". Therein the soil nutrient tester [4] had been put into the full production. Moreover the foundation on the expert systems would not research in this paper including the fertilizer recipes and fertilization model with the different crops, geographical location and climate varies [5-9].

Presently, the research of field information rapid acquisition technology is still far behind the other technologies in support of precision agriculture, and it had become an important issue on the international related research field. The keys on technological innovation are the fast real-time survey method in order to increase the sampling density, and find new sensor technology to meet the actual production requirements or even further improvements [2]. Author firstly overviewed three areas development of PFMIS on data collection, data processing and information management systems based on GPS (Global Positioning System) and GIS (Geographic Information System) technologies, secondly designed the PFMIS framework with the new technical methods, thirdly its advantages and feasibility were analyzed.

\section{The Development of Precision Fertilization Based on GPS and GIS}

Precision fertilization agriculture technologies are generally the agriculture management information system [10], which can capture quickly and efficiently and describe the spatial information of the variable environment impacted on crop growth, and are important to carry out the precision agriculture practice. The spatial coordinates and their varieties attribute information of soil resources were obtained after collecting data. Then data-processing technology and information management systems of attribute information based on spatial coordinates are keys.

\subsection{The Spatial Data Surveying Technique of PFMIS}

RS (Remote Sensing) and GPS single point positioning were the popular surveying modes on the spatial data acquisition $[11,12]$. RS technology was one of main fielddata sources to precision agriculture, which collect the ground spectral reflectance features used relative high-resolution-rate sensors, to monitor comprehensively in different crop growing period, in order to provide a large number of the space-time 
change information in farming field. Then the analyses of spatial characterization and location were finished based on the spectral information [13]. The time-series images, obtained by RS, were widely applied on the large-scale agricultural yield estimation. Because of some shortages such as the low-resolution rate spatial information, data post-processing, related support needs and lack of infrastructure, and so on, now GPS was used to make up the soil samples collected and fertilizer operations in positioning and navigation. GPS single point positioning can collect spatial information on kinematic real-time, all-weather, and convenient mode. Only one GPS receiver was used to arrive to the positioning accuracy in the $10 \mathrm{~m}$ level. But the accuracy level can not meet the requirements of the precise agriculture, while time, some shortages of high cost, low efficiency and poor real-time on the updating database; Otherwise DGPS (differential GPS) requires less than 2 receivers, one for the base-station, others for the mobile-stations, which must keep distance to base-station within $20 \mathrm{~km}$, and the code observations can be achieved [14-16], which is a large farm operations is limited.

With the development of CORS established more and more in the region, and in different fields, the RTK positioning and navigation realized conveniently in the centimeter-level precision used the network RTK technology on at least one receiver [15-19]. This paper designed the spatial data - soil resources information and data acquisition subsystem by GPS CORS technology, and improved the positioning accuracy and stability by accordingly data processing and software development.

\subsection{Data Processing Method of the Soil Resource Information}

After data collection, a variety of attribute information on spatial coordinates data were accessed: The physical and chemical properties of soil include soil types, soil texture, organic matter and soil $\mathrm{pH}$, and other data. Soil nutrient data include soil nitrogen, phosphorus, potassium and other large number of nutrients and available nutrient data, calcium, magnesium, sulfur, chlorine, zinc, manganese, copper, iron, molybdenum and boron and other micronutrients data are included [6]. The key issue is how to process the data transformation from point-to-surface of the soil resource attribute data. The spatial interpolation methods were adopted on data-processing, mainly the Kriging geo-statistical interpolation and inversely proportional to distance interpolation method. On the condition of the same sampling density, in descending order of interpolation precision is plains, post area, hills, mountains, that is, with the complexity increased of the terrain interpolated, the accuracy was reduced. While time the sampling density increased more on the hills, mountains, etc., and the accuracy of the results were improved more [20]. Compare with the neural network methods (NN), as the sampling density is high, the same accuracy of the results of Kriging and inversely proportional to distance interpolation methods was obtained; but the case of low sampling density, the results of neural networks were the better accuracy.

Mueller T.G. and so on [21] experimented on the impact of soil fertility distribution to the different grid sampling density and interpolation methods. They selected the three fields, the different sampling plan were made according to the different sampling instance points and the different interpolation of Kriging and inversely proportional to 
distance methods. They drew the different attribute the spatial distribution of the soil available $\mathrm{P}, \mathrm{K}$ and $\mathrm{PH}$ value, and calculated the standard deviation values of the above interpolation results. The results showed that Kriging interpolation accuracy is different for the soil available P, K and PH in the different plots of the same kind of soil properties. And in the same plot, the available $\mathrm{P}$ of different sampling interval of Kriging results indicated that the higher sampling density, the higher the interpolation accuracy.

In summary, the spatial variability laws of the agricultural soil information have their own characteristics. And the qualitative and quantitative relationship between crop production and soil properties on the spatial distribution was reported rarely. So the spatial heterogeneity of the soil information under certain conditions still needs further study. The artificial neural network (ANN) is a self-adaptive method of mapping, and do not make any assumptions in data processing. ANN is reasonable in theory, and be able to avoid the unknown factors, to reduce the model error. So ANN's results are high accuracy. The spatial interpolation accuracy based ANN are higher and more stable than the usual variety of data interpolation methods, and the fewer number of sample points are required, the results can be directly obtained, the loss of accuracy are reduced in the middle link. Therefore, the rational design of NN can be efficient to solve the fertilization test zone on the condition of the less known points.

\subsection{Management Information Platform on GIS}

GIS are computer software platforms used for input, storage, retrieval, analysis, processing and presentation of geospatial data. And it associated with each other coordinates on the same location values based the geo-spatial database of the geographic characteristics. GIS is the brain of precision agriculture. And GIS firstly deposited the advance decision-making system, such as expert systems, and the data processing module, then received the collected information from the real-time sensor on variable rate fertilizer and the monitoring systems (GPS, RS, etc.), thirdly showed and mapped electronic pictures on unified coordinate system for these data through organization, processing, statistical analysis, finally made intelligent decisions through the expert system and achieve adjust the amount of inputs or operations used automatic controller-executer equipment [13]. Domain is the computer jargon of the functional domain covered with group systems on the similar needs or software applications. Domain engineering is the procedure that certain a system of domain application was analyzed, the common and variability of demands was refined, the domain models were made, and the architectures were designed, then the reusable components of domain were developed and organized. Domain engineering includes several parts of domain analysis, design, implementation and spread out. Domain engineering provides a strong support to software reuse, which helps to produce components with high reusability, and its product is the domain analysis model and domain-specific software architecture $[22,23]$. In this part, authors make a guide use the knowledge and theory of the domain engineering, software components, design patterns and 
others, and utilize the ESRI's ArcGIS Engine as the platform for the needs of GIS application functional modules were met in of GIS application development component, and the developers only focused on the specific business development of the module, to avoid duplication of functions of development on based GIS applications, so as to improve the development efficiency, reduce the development costs. After the function of operational modules has been refined and standardized, they can be added to the component library used component management tools, for use the other similar GIS application system development in future [24-26].

In the practice of precision agriculture, GIS is mainly used for the establishment of agricultural land management, the development trend of soil data, natural conditions, production conditions, the seedlings crop growth and insect pest, and the geographic statistical processing, graphics conversion and expression of crop yields and other spatial information, in order to offer prescription scheme of the analysis of spatial differences and implementation of control [27, 28]. The multi-layer maps of farmland yield and spatial information were firstly generated on GIS. Then the decision-makers analyzed the causes, make a diagnosis, and provide scientific prescription based on yield-spatial differences with crop production management decision support systems and expert intelligent management systems. Thirdly the field crop management prescription maps were produced on GIS, and zoning regulation implemented to guide the scientific operations. When the agricultural geography spatial information map were produced, how to define the sampling density, sampling costs and dataprocessing methods, satisfied to more accurately reflect the spatial distribution of parameters, is still next-step study [2].

\section{Design of PFMIS Based on CORS and GIS}

As known, two and above GPS receivers are used on DGPS to collect the spatial information on fields. However, with the regional CORS (Continuously Operational Reference System) widely development in the domestic, only one GPS receiver can be finished the spatial data of large-scale, all-weather, high-precision, kinematic real-time observation on CORS, which make up for the shortages of RS, such as low-precision of spatial information, time-delay of collection. The accuracy, reliability and real-time characters of spatial data were improved on CORS technology.

3-dimensional spatial coordinate information on GPS and soil resources attribute information on soil nutrient testing device were obtained to found the fields database, and the thematic maps of crop growth environment and yield spatial distribution were generated in real time or near real-time, in order to update the achievement on current trend. Then these maps overlay with other soil elements maps based spatialconsistency to analysis. Finally the corresponding mathematical models were founded to provide the basis of the soil fine fertilization.

Based GIS platform, the spatial data grid maps of soil resource were re-developed on ArcGIS Engine, including of some management information functions, such as 
analysis, queries, additions and deletions, updates, and contrast of the collected soil resource data. Then the basis on decision making was provided to variable-rate fertilization with the expert systems. WebGIS system were built with ArcGIS Server to complete the web publishing or query information of precision fertilization, and the efficient fertilization programs and crop fertilizer criteria were recommended for users in order to achieve the modern agricultural production patterns and the technology system of the higher yield, quality and level, and arrive to high efficiency, savingenergy, high production, environmental protection.

\subsection{Technique of PFMIS on CORS and GIS}

According to the Shandong Province's actual situation of agriculture, soil and climate, the research of application of technology of data collection, data processing and management information system queries were carried out on the soil resource information of PFMIS. And PFMIS were designed aimed to reducing the investment on crop early growth, low waste in the growth-medium, increasing the profits the later, and reducing the environmental pollution in the long term. When PFMIS were established based on GPS and GIS technology. The farmland spatial information were collected using GPS, and the accuracy, reliability and real-time of the spatial data were improved with CORS technology. The keys on data standardization technology were researched, and the spatial grid distribution maps of soil nutrients and crop yield were produced by GIS re-development on soil topics, some functions of web publishing and query were realized to provide the basis on decision making, above as shown in Fig. 1, including:

(1) To argue the scheme design of CORS of the geographic information acquisition subsystem of PFMIS;

(2) To model on the from-point-to-surface data processing of soil resources information and contour of soil resources property information, such as geostatistical Kriging, surface fitting, ANN and each other algorithms comparison;

(3) To realize the standardization of data processing methods on the soil resource information, including the undulating terrain classification, sampling interval grading, sample units install, etc., and achieve the layover and analysis on multi-characterlayers of a variety of space-based information.

(4) To determine the communication and query tool on the Web-platform of PFMIS, to interactive services, including synchronous communication: IRC, ICQ, MSN, Skype; and asynchronous communication: E-mail, Mailing list and so on.

\subsection{Design of PFMIS on CORS and GIS}

Research procedures and data-process flow shown in Fig. 2, including:

(1) Design the collection subsystem of PFMIS on CORS technology. As a large areas of farmland, a long time measurement, mobile frequently, basically covered by CORS of Shandong Province, to prove the scheme of network distribution and the feasibility of cm-level accuracy. A GPS receiver was used to observe and receive realtime differential signal, and cm-level positioning was obtained by data processing. The 
agricultural nutrient tester, temperature-hygrometer and others were used to collect the soil resources information samplings. To ensure the accuracy of those, the sorts data were input GIS platform to manage, the kinematic real-time acquisition subsystem of the spatial and soil attribute information of PFMIS was completed. And this subsystem provided these real-time or near real-time various soil-attribute layer to analysis on the follow-up GIS applications.

(2) Re-development of the scheme GIS based on ArcGIS Engine. To research the key technologies of data standardization, mainly attribute data standardization and a variety of data analysis and processing, including geostatistics, neural networks, genetic algorithms, to generate DEM, soil resources, nutrients (N, P, K, Ca, etc.), temperature, humidity and yield, etc. Attribute distribution maps, then various types of thematic maps were produced by the spatial analysis and mapping capabilities of GIS, through spatial overlay analysis of land resources. Next-step the variable fertilizer distribution maps were generated together with expert knowledge. It is keys to develop GIS analysis component library on this stage of system design.

(3) To renew collecting data and update the variable fertilizer maps with the growth of crops in different time periods. The network applications of PFMIS were achieved by Web GIS, including the dissemination of information, interactive inquiry service, and so on.

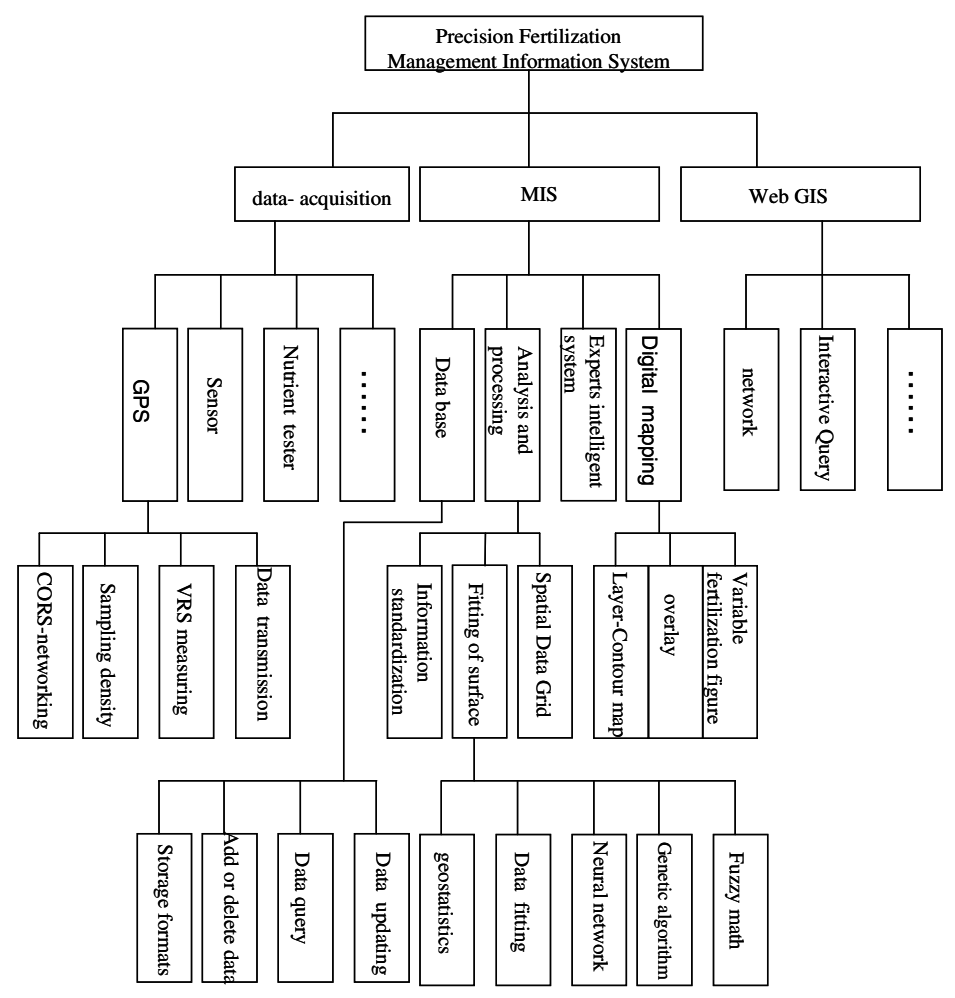

Fig. 1. The Design Structure of PFMIS 


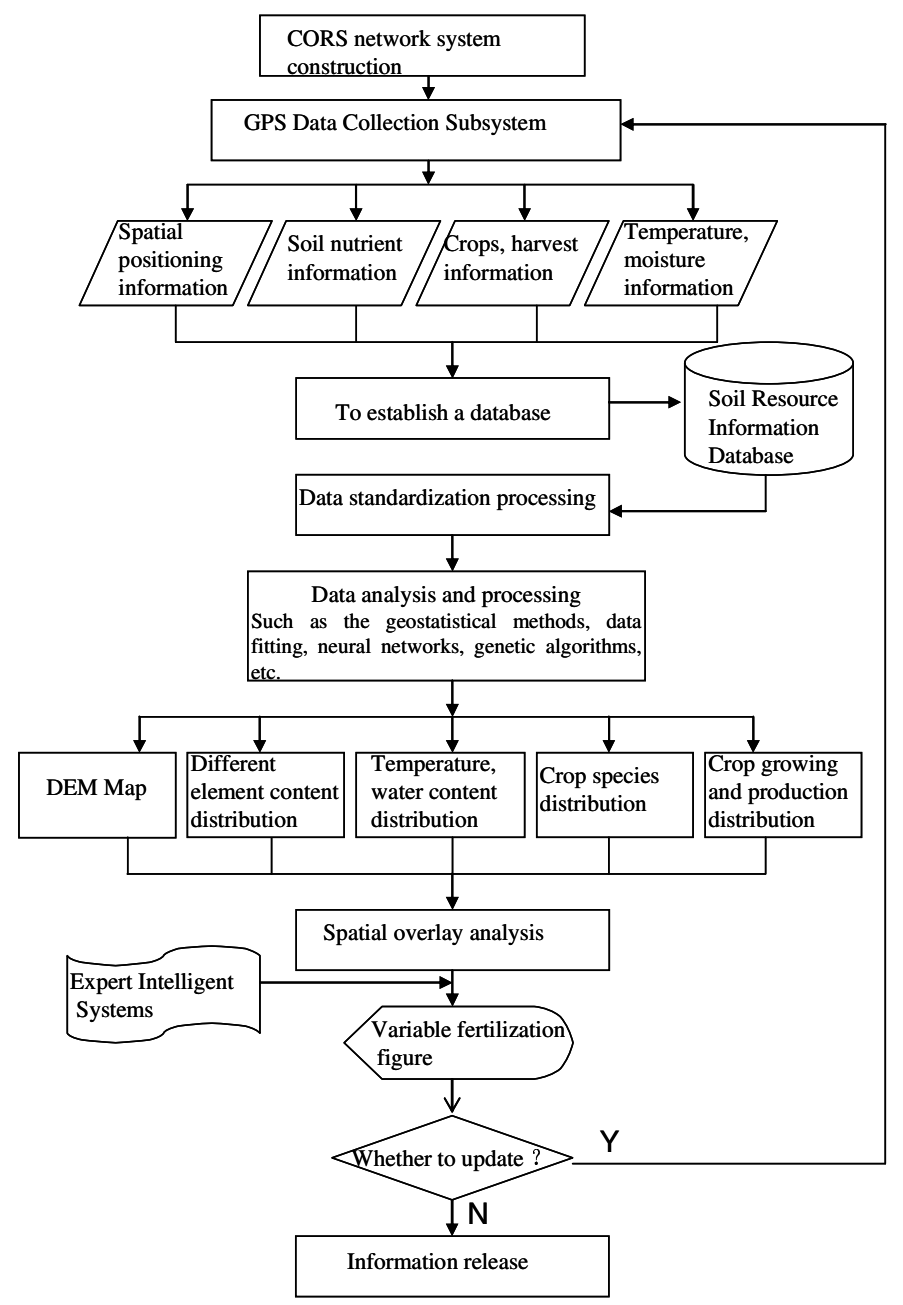

Fig. 2. The processing flow of PFMIS

\section{Conclusions}

Precision agriculture is an inevitable trend on the agriculture development from the coarse type to the fine. The research on the high-precise spatial data real-time collection, data processing and management information system technology is presently one of focuses on precision agriculture. On the topics the key technologies include: firstly the achievements of real-time data collection and processing were obtained used CORS technology, and whose accuracy levels would be better than $10 \mathrm{~cm}$; secondly the models of data processing and analysis of soil attribute and crop yield on spatial data were founded based on multi-intelligence computing algorithms; thirdly the re-developments of the soil-thematic GIS on ArcGIS Engine were run. While, how to define the sampling density, sampling costs and data-processing 
methods, to more accurately reflect the spatial distribution of parameters, is worth of deeply study.

Acknowledgments. This work is supported by National natural science foundation of China (No. 40704001, 40876051).

\section{References}

1. Edan, Y., Han, S., Kondo, N.: Automation in Agriculture. Springer Handbook of Automation, Part G, pp. 1095-1128 (2009)

2. Wang, M.: Development of precise agriculture and innovation of engineering technology. Transaction of Agriculture Engineering 15(1), 1-8 (1999) (in Chinese)

3. Wang, M.: Thinking Through the Experiment, Demonstration and Development Research on Precision Agriculture. Review of China Agricultural Science and Technology 5(1), 7-12 (2003) (in Chinese)

4. http: //china.toocle.com

5. Chen, Z., Wei, L., Lin, Q.: Design and implementation of precision rubber fertilization information system based on Web GIS. Chinese Agricultural Science Bulletin 24(7), 473-477 (2008) (in Chinese)

6. Chen, W., Hua, Y., Zhang, S.: Tobacco Precision Fertilization Formula Information System Based on GIS. Journal of Geomatics Science and Technology 25(6), 455-458 (2008) (in Chinese)

7. Sun, Z., Zhang, X., Jiang, W., et al.: Design and realization of vegetable precise fertilization expert system. Agriculture Network Information (8), 15-17 (2005) (in Chinese)

8. Wang, Q., Ma, Y., Hu, H., et al.: The Research on the Method of Precision Fertilization Based on GIS and GPS in China. Chinese Agricultural Science Bulletin (7) (2006) (in Chinese)

9. Xu, Y., Ni, M., Liu, G., et al.: The Pre-Investigation of Precision Fertilization and Expert Consultation System. Tibet Journal of Agricultural Sciences (3), 31-36 (2009) (in Chinese)

10. Blackmore, B.S.: Precision Agriculture: an introduction. Outlook Agric. 23(4) (1994)

11. Lu, J., Guo, J.: Application of GPS in Precision Agriculture. Geotichnical Investigation and Surveying (1), 50-51 (2002) (in Chinese)

12. Zhang, S.: The Study of the Collection and Process of Field Information of Precision Agriculture Based on the GPS and GIS. University of Zhejiang PhD (2003) (in Chinese)

13. Wang, C., Niu, Z., Tang, H.: The earth observation technology and precision agriculture. Science Press, Beijing (2001) (in Chinese)

14. Zhou, Z., Yi, J., Zhou, Q.: Theory and application of GPS satellite surveying (Revision). Surveying and Mapping Press, Beijing (1997) (in Chinese)

15. Hofmann-Wellenhof, B., Lichtenegger, H., Collins, J.: GPS Theory and practice, 5th revised edn. Springer, Wien (2001)

16. Xu, G.: GPS Theory, Algorithms and Applications. Springer, Berlin (2003)

17. Dang, Y., Bei, J., Cheng, Y.: Principles and applications of global navigation satellite system. Surveying and Mapping Press, Beijing (2007) (in Chinese)

18. Huang, D., Xiong, Y., Yuan, L.: Theory and Practice of Global Positioning System. Southwest Jiaotong University Press, Chendu (2006) (in Chinese) 
19. Tang, W., Sun, H., Liu, J.: Ambiguity resolution of single epoch single frequency data with baseline length constraint using LAMBDA algorithm. Geomatics and information science of Wuhan University 30(5), 444-446 (2005) (in Chinese)

20. Lei, N., Wang, X., Jiang, J., et al.: Comparison of Kriging interpolation precisions in different topographical units and number of samples:a case study of spatial distribution of soil total nitrogen in the Shucheng County. Hydrogeology \& Engineering Geology (5), 86-91 (2008) (in Chinese)

21. Mueller, T.G., Wells, K.L., Thomas, G.W., et al.: Soil fertility map quality: case studies in Kentucky. In: Proceedings of Fifth International Conference on Proceedings Agriculture (CD), USA (2000)

22. Ren, J., Lv, G., Wang, Q.: Research on the Integration of Geography Information System and Model in Multiple Tier System. Acta Geodaetica Et Cartographic Sinica 32(2), 178-182 (2003) (in Chinese)

23. Wang, J., Bao, S., Yu, Y., et al.: Realization of geological section map model based GIS template. Science of Surveying and Mapping 33(5), 184-186 (2008) (in Chinese)

24. Liu, Z., Meng, Y., Yin, A.: Design and Realization of ArcGIS Engine Component. Geospatial Information (1), 43-47 (2008) (in Chinese)

25. Xiong, W., Liu, Z., Wang, S.: Design and Realization of the Cartography Template Management System. Geospatial Information (2), 90-92 (2010) (in Chinese)

26. Zhang, Z., Yang, J.: Transport planning information system based on Arc GIS. Science of Surveying and Mapping 30(1), 86-89 (2005) (in Chinese)

27. Runquist, S., Zhang, N., Taylor, R.: Development a field-level geographic information system. Computers and Electronics in Agriculture 31, 201-209 (2001)

28. Zhang, N., Taylor, R.: Applications of a field-level geographic information system(FIS) in precession agriculture. In: Proceedings of Fifth International Conference on Precision Agriculture (CD), USA (2000) 